\title{
Effect of Low-Level Laser Irradiation on Accommodation and Visual Fatigue
}

\author{
Ching-Huang Lin' \\ Hsien-Chang Lin ${ }^{2}$ \\ Chien-Yu Chen ${ }^{3}$ \\ Chong-Chung Lih ${ }^{4}$ \\ 'Department of Electronic Engineering, \\ National Yunlin University of Science \& \\ Technology, Yunlin, 640, Taiwan; \\ ${ }^{2}$ Graduate School of Engineering Science \\ and Technology, National Yunlin \\ University of Science \& Technology, \\ Yunlin, 640, Taiwan; ${ }^{3}$ Graduate Institute \\ of Color and Illumination Technology, \\ National Taiwan University of Science and \\ Technology, Taipei, 106, Taiwan; \\ ${ }^{4}$ Department of Optometry, Central \\ Taiwan University of Science and \\ Technology, Taichung, 406, Taiwan
}

Purpose: This study used low-level laser irradiation on auricular points for the eyes of junior college teenagers with myopia to observe the changes in refractive errors and eye accommodation. According to the Sterner's study, we used the relationship between the amplitude and subjective symptoms to assess the degree of improvement in visual fatigue caused by changes in accommodation.

Methods: Participants were 49 healthy junior college teenagers, with an average age of $16.04 \pm 0.80$ years old, and the refractive errors of both eyes were between $-1.50 \mathrm{D}$ and -5.00 D. All participants underwent refractive error tests before and after class and after a 30-min break. In a 30-min break, all participants underwent two tests of with (experimental group) and without (control group) low-level laser irradiation on the auricular points. Then, refractive error tests were conducted to evaluate the participants' eye accommodation.

Results: The results revealed that with treatment of the auricular points with and without low-level laser irradiation, the refractive error of the participants' binocular left and right eyes decreased by $-0.12 \mathrm{D}(-0.18 \mathrm{D})$ in the experimental grou and by $-0.05 \mathrm{D}(-0.02 \mathrm{D})$ in the control group. For the amplitudes of accommodation (AA) examination, the AA of the participants' binocular in the experimental group became $7.98 \pm 1.35 \mathrm{D}(7.65 \pm 1.37 \mathrm{D})$. The AA of participants' binocular can recover to the level before the class.

Conclusion: The results indicated that low-level laser irradiation at auricular points restored the eye accommodation faster than did natural closing of the eyes.

Keywords: low-level laser irradiation, myopia, teenager, accommodation, auricular point

\section{Introduction}

A low-level laser has an output power less than $100 \mathrm{~mW}$. This power is too low to destroy the bonding (hydrogen bond and van der Waals force) between molecules in tissues. Therefore, low-power irradiation does not change molecular structures or cause protein denaturation and apoptosis. Low-level lasers provide adequate energy to stimulate cells and induce or strengthen certain biological responses, such as promotion of local blood circulation, moderation of cellular functions, improvement of immune functions, and improvement of cellular metabolism and proliferation abilities. The Laser acupuncture (LA) uses laser beam instead of acupuncture needles to irradiate acupoints according to traditional Chinese medicine acupuncture principles. This method can improve or alleviate conditions and pain. Lowlevel laser irradiation is a type of phototherapy. ${ }^{1}$ A Hungarian scholar named Mester proposed in 1966 that low-level irradiation exerts biostimulation effects ${ }^{2}$. Since then, laser technology has been progressively developed and widely used. Advanced laser technology has been widely applied in combination with traditional
Correspondence: Chong-Chung Lih Department of Optometry, Centra Taiwan University of Science and Technology, Taichung, 406, Taiwan Tel +866 422391647 698I

Email cclih@phys.nthu.edu.tw 
acupuncture techniques. This has led to the invention of laser acupuncture (LA), which involves the use of lowpower laser beams to directly irradiate or concentrate or expand the beams to irradiate the surface of or through acupuncture points, thereby effectively stimulating the acupuncture point. Subsequently, the meridians can be dredged, viscera accommodated, qi and blood invigorated, and yin and yang balanced to strengthen vital qi and eliminate pathogenic factors as well as treat diseases and maintain overall health. Body parts that are extremely sensitive to stimulation are usually chosen as acupuncture points. These points contain numerous sensors that are closely connected to surrounding muscle tissues, nerves, blood vessels, and lymph vessels. When a laser beam is applied to acupuncture points, it penetrates the skin surface with the needlestick characteristics. Approximately $40 \%$ of laser energy is absorbed by the skin and converted into heat, thus functioning as moxibustion. Therefore, laser stimulation can be used for both acupuncture and moxibustion. ${ }^{3,4}$ Compared with traditional acupuncture methods, LA is more advantageous because it is painless, sterile, safe, easy to control, and adjustable and provides therapeutic effects of acupuncture and moxibustion. Therefore, patients are readily willing to use such a therapy method. In addition to acupuncture and moxibustion effects, laser irradiation on acupuncture points presents multiple unique advantages. Therefore, the use of laser technology has attracted considerable academic attention and numerous clinical applications. ${ }^{5,6}$

The auricular points (Figure 1) are acupoints distributed on the auricle; they are also called trigger points or stimulation points. In visceral or somatic diseases, people often experience local reactions, such as tenderness and color changes, at specific parts on the auricle. This phenomenon can be used as a reference for disease diagnosis. In addition, stimulation of auricular points can have certain regulatory effects on corresponding viscera, maintain the body's internal functional balance, alleviate ciliary muscle spasm, and improve accommodation of eyes. Some studies have mostly used auricular points to treat myopia. ${ }^{7-14}$ For example, $\mathrm{Li}^{13}{ }^{13}$ in 1988 , used intradermal needle therapy on auricular points for myopia treatment in 416 patients, totaling 812 eyes. A total of 521 eyes significantly improved, 240 eyes nonsignificantly improved, and 48 eyes did not show improvement. The total effective rate was $94.1 \%$. Liu, ${ }^{14}$ in 2012 , used 140 cases (256 eyes) of adolescent patients with mild myopia and randomly divided them into a treatment group (70 cases, 126 eyes)

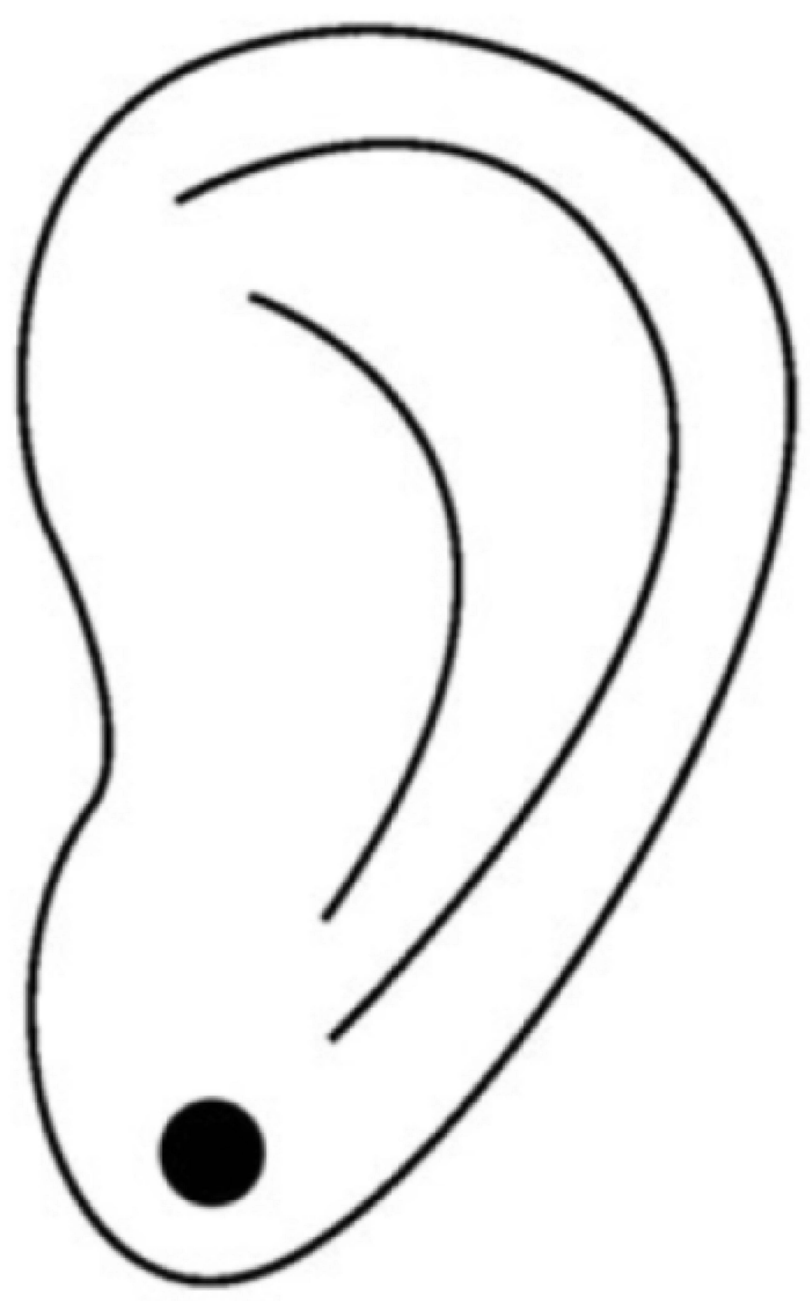

Figure I Auricular acupuncture points.

and a control group (70 cases, 130 eyes). The treatment group used sticking ear points. The control group received compound topiramate eye drops once every night before going to bed. After 6 weeks, the visual acuity changes were observed with the international standard visual acuity chart. The result is that the visual acuity of the pressing ear acupoint group is significantly improved after treatment, and the curative effect is better than that of the control group. The total effective rates of the two groups were $83.33 \%$ and $62.07 \%$, respectively. There was a significant difference between the two groups $(\mathrm{P}<0.01)$. Therefore, Liu argued that auricular therapy can be effective through nerve conduction. During auricular acupressure, the nerves widely distributed around the auricle conduct upward stimulation through impulse and indirectly or directly moderate the refractive system of the eyes.

Asthenopia refers to the eye fatigue caused by overuse of the eyes when engaged in close-gaze work or study. ${ }^{15-18}$ The 
clinical manifestations are mostly of view that the vision cannot be sustained, the vision of the long-sighted person is $\mathrm{dim}$, and the eyeball or periorbital soreness is felt. Jaschinski $\mathrm{Kruza}^{17}$ pointed out that ciliary body adjustment ability is related to visual fatigue. The Satio ${ }^{18}$ study also pointed out that the cause of visual fatigue caused by visual work is the temporary change of accommodation. For young people, reading at school all day or using $3 \mathrm{C}$ products for a long time will cause young people to overuse their eyes, which will lead to an increase in myopia and eye-related diseases. From Bertils' study, ${ }^{19}$ the amplitude of accommodation has the potential to assess the degree of visual fatigue ability. This study used low-level laser irradiation on acupuncture points to achieve the effect of acupuncture and moxibustion in traditional Chinese medicine. The auricle points for the eyes were irradiated to observe the changes in refractive errors and eye accommodation. At the same time, we hope to quickly relieve visual fatigue caused by adjustment changes.

\section{Experimental Design Participants}

Participants were 49 healthy junior college teenagers. The male to female ratio was 20:29, and the mean age was 16.04 \pm 0.80 years. The visual acuity of the left and right eyes of the participants was corrected to 1.0, with the refractive error between $-1.50 \mathrm{D}$ and $-5.00 \mathrm{D}$. The anterior region and fundus of the participants' eyes were examined to rule out organic diseases. Each participant was required to get ample sleep and avoid activities that may cause eye strain before the experiment. They were also required to avoid caffeine and alcohol 8 hours before the experiment. Individuals with a history of eye diseases within 6 months prior to the experiment were excluded. All participants understood the experimental procedure of the present study and consented to participate in the experiment.

\section{Experimental Environment}

To facilitate dioptric power examination before and after class and after a 30-min break, a bright room was selected as the experiment site. The room temperature was controlled at $25 \pm 2^{\circ} \mathrm{C}^{17}$ and room illumination at 200-400 lx. The experimental environment is shown in Figure 2.

\section{Experimental Equipment}

The laser equipment adopts a power of $30 \mathrm{~mW}$, wavelength of $830 \mathrm{~nm}$, and a frequency of $7 \mathrm{~Hz}$. The irradiation

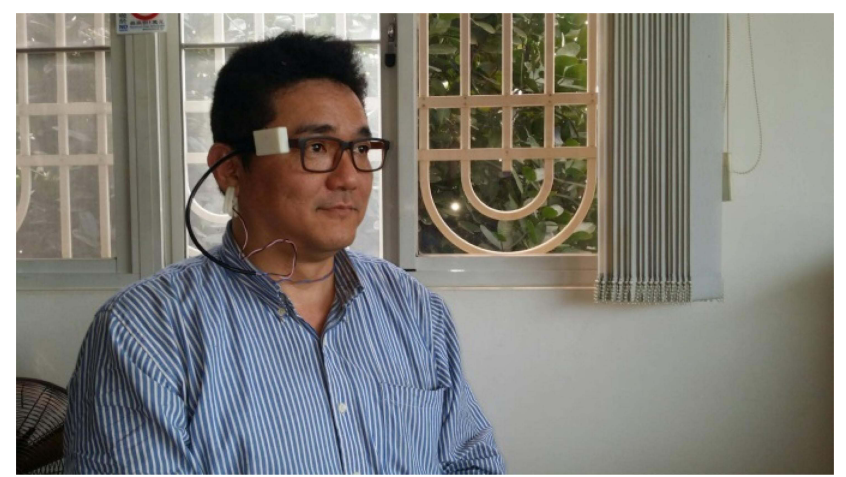

Figure 2 Experimental environment.

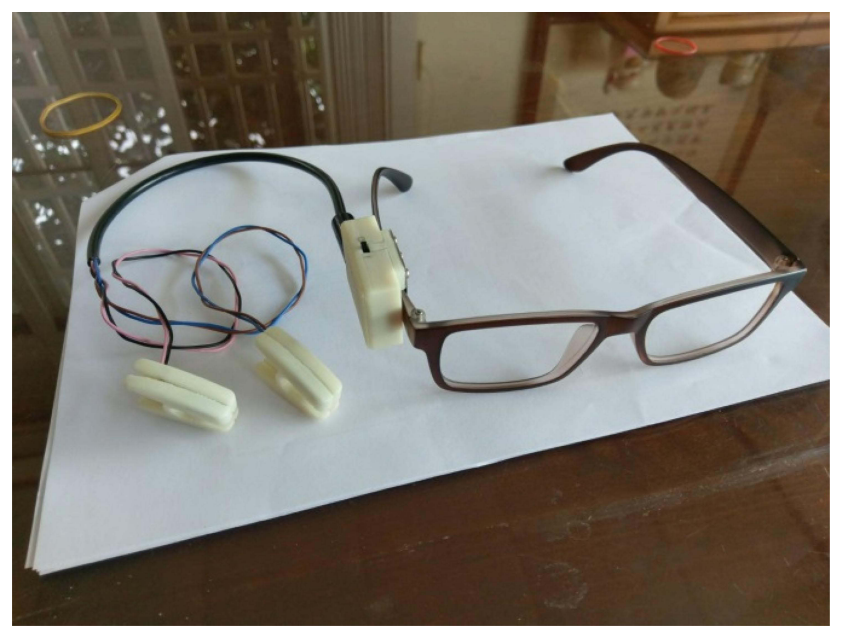

Figure 3 Experimental equipment.

time was set to be $30 \mathrm{~min}$. The diameter of the laser beam was $0.1 \mathrm{~cm}^{2}$. The laser equipment is shown in Figure 3. Optometry equipment adopts SHIN-NIPPON K5001 autorefractor.

\section{Experimental Method}

This experiment was divided into two runs and one week apart each time. In the beginning, the participants underwent dioptric power tests before class, after class, and after a 30min break. This is the result of the control group. In the second run, the participants underwent dioptric power tests before class, after class, and after low-level laser irradiation on the auricular points for $30 \mathrm{~min}$ after class. This is the result of the experimental group. These two runs experimental procedures are shown in Figures 4 and 5. All participants are only active in school during the experiment. 


\section{Start}

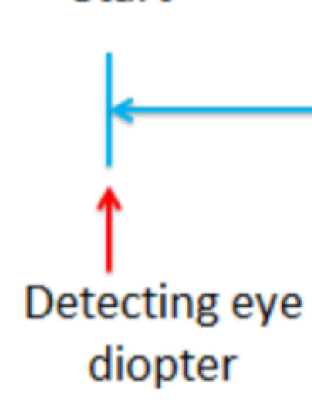

End

rest

class

9hours

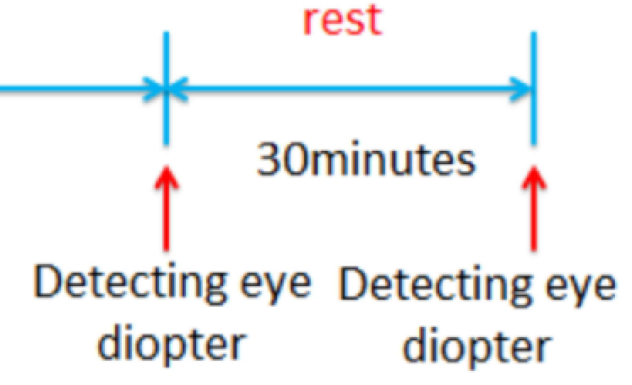

Figure 4 Experimental flow of control group.

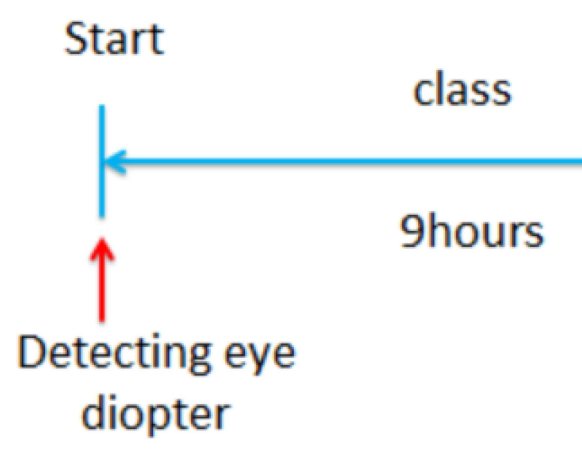

Figure 5 Experimental flow of experimental group.

Any time during the experiment, if a participant felt uneasy, had difficulty in breathing, exhibited paleness or experienced dizziness or vertigo because of the laser irradiation, or if the participant wanted to terminate the experiment, they were allowed to stop the experiment immediately.

\section{Statistical Analysis}

This variance of dioptric power was analyzed using SPSS. Paired-sample $t$-tests were conducted on the participants' dioptric power data before and after class and with and without low-level laser irradiation. The level of significance was set to be 0.05 .

\section{Results}

\section{Refractive Error Examination}

The results of the control group and experimental group in the refractive error examination are shown in Table 1. In control group, the refractive error variations in the left and right eyes of the participants before and after class and after the 30-min break are shown in Figure 6. The refractive error in both eyes after class increased compared with those before class, suggesting that the teenagers' eyes presented visual fatigue with increased refractive error after they attended class for $9 \mathrm{~h}$. After the 30-min break after class during which time the students closed and rested their eyes,

Table I Refractive Error of the Control and Experimental Group

\begin{tabular}{|l|l|l|l|l|}
\hline Control Group & Before Class & After Class & After a 30-min Rest with Eyes Closed & P-value \\
\hline $\begin{array}{l}\text { Right eye refractive error } \\
\text { Left eye refractive error }\end{array}$ & $-2.78 \pm 1.77$ & $-2.85 \pm 1.73$ & $-2.83 \pm 1.80$ & $-2.49 \pm 1.75$ \\
\hline Experimental Group & Before Class & After Class & After Low-Level Laser Irradiation for 30 min & $P>05$ \\
\hline Right eye refractive error & $-2.82 \pm 1.80$ & $-2.94 \pm 1.77$ & $-2.76 \pm 1.78$ & \\
Left eye refractive error & $-2.44 \pm 1.75$ & $-2.52 \pm 1.83$ & $-2.40 \pm 1.75$ & $P<0.05$ \\
\hline
\end{tabular}




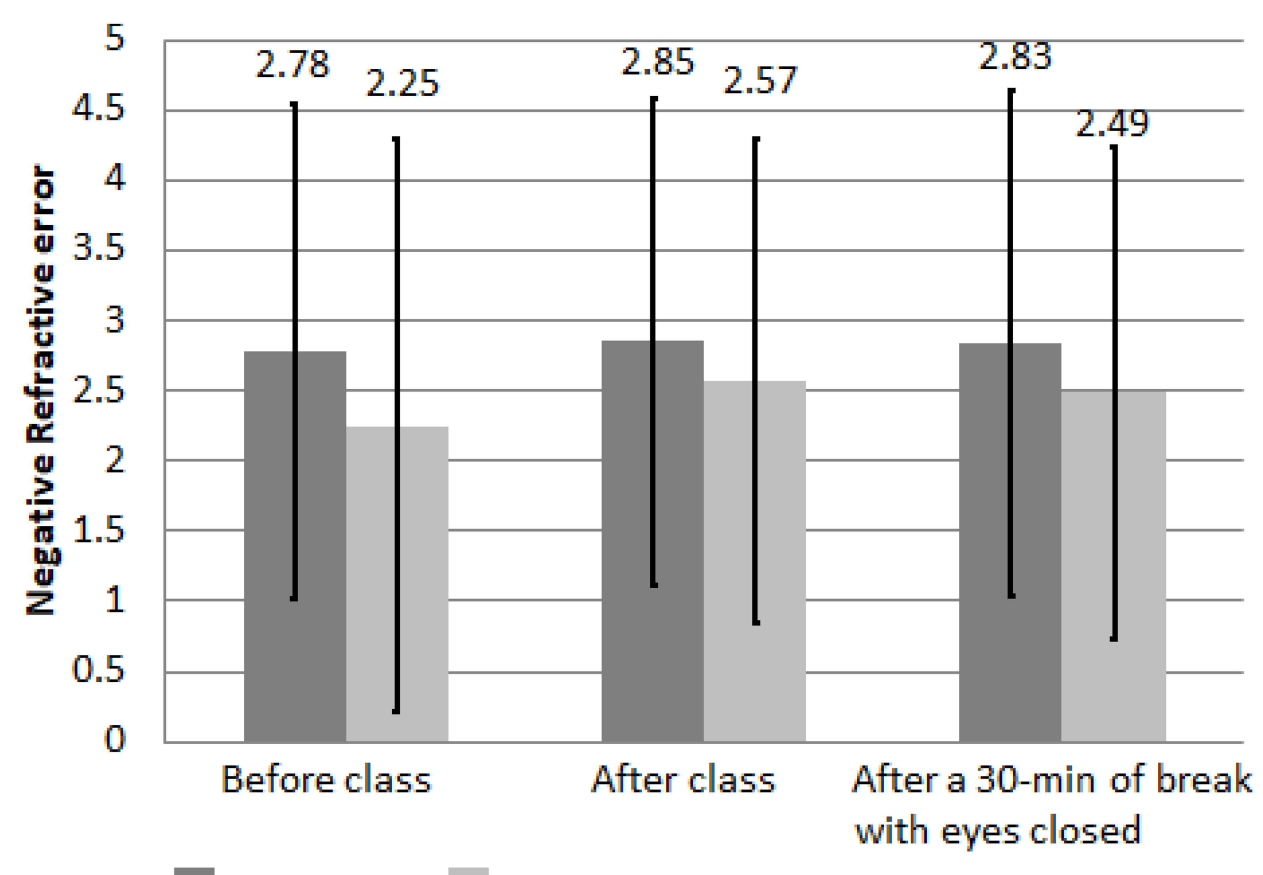

\section{RIGHT EYE LEFT EYE}

Figure 6 Refractive error before and after class and after a 30-min break in the control group.

the refractive error in their eyes decreased compared with the values immediately after class. These results indicated that the participants' eye refractive error decreased after a 30-min rest with eyes closed.
In the experimental group, the refractive error changes in the left and right eyes of the participants before and after class and after low-level laser irradiation on the auricular points for $30 \mathrm{~min}$ after class are shown in

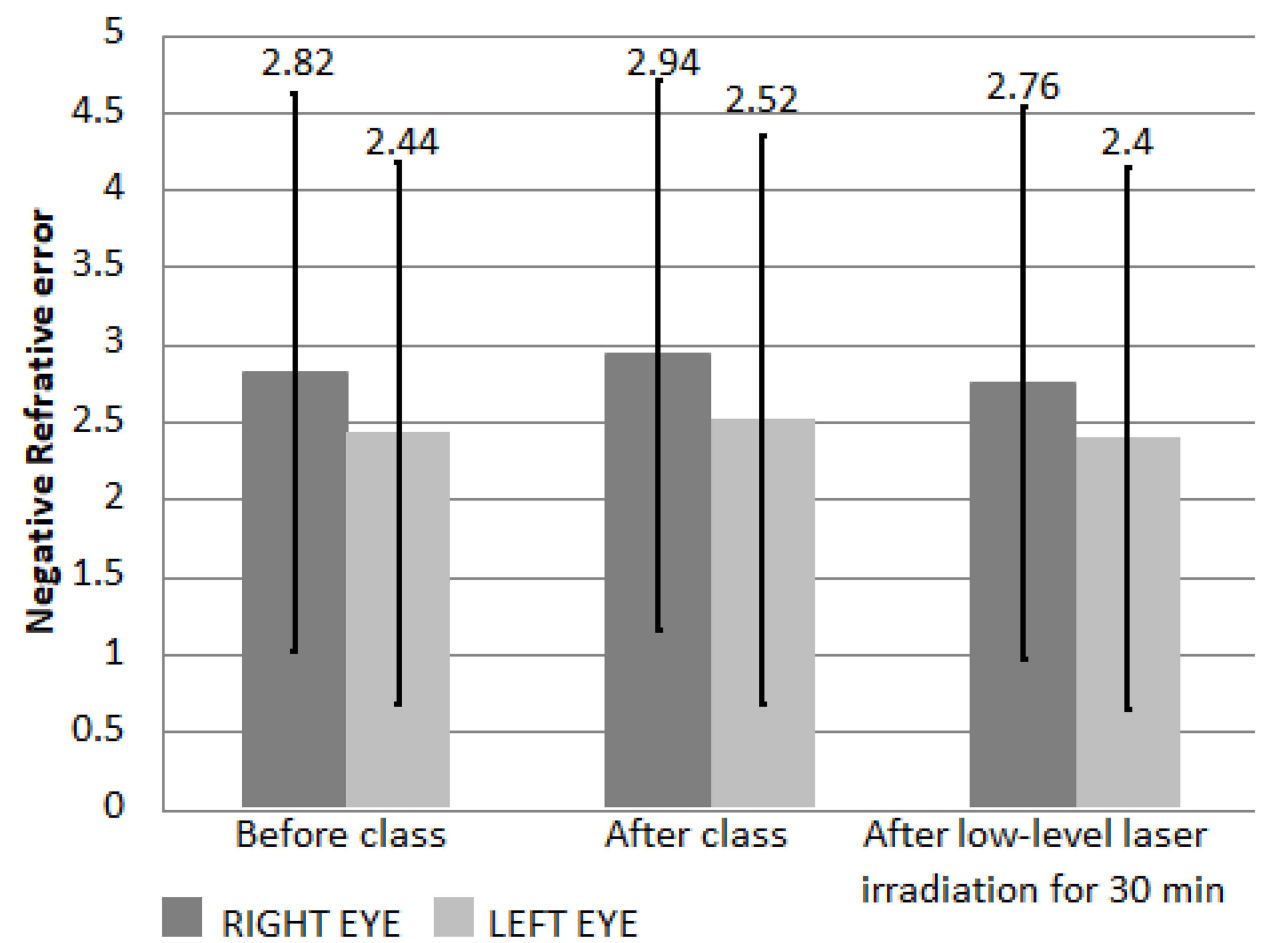

Figure 7 Refractive error before and after class and after $30 \mathrm{~min}$ of low-level laser irradiation in the experimental group. 
Table $2 \mathrm{AA}$ of the Control and Experimental Group

Unit: D

\begin{tabular}{|l|l|l|l|l|}
\hline Control Group & Before Class & After Class & After a 30-min Rest with Eyes Closed & P-value \\
\hline $\begin{array}{l}\text { Right eye AA } \\
\text { Left eye AA }\end{array}$ & $7.63 \pm 1.33$ & $5.53 \pm 1.34$ & $6.58 \pm 1.38$ & P \\
\hline $8.12 \pm 1.69$ & $5.77 \pm 1.31$ & 0.05 \\
\hline Experimental Group & Before Class & After Class & After Low-Level Laser Irradiation for 30 min & \\
\hline Right eye AA & $7.60 \pm 1.37$ & $5.53 \pm 1.39$ & $7.65 \pm 1.37$ & \\
Left eye AA & $7.93 \pm 1.31$ & $5.84 \pm 1.43$ & $7.98 \pm 1.35$ & $P<0.05$ \\
\hline
\end{tabular}

Figure 7. The refractive error in both eyes increased, indicating that the participants presented visual fatigue in both eyes with increases in the refractive error after attending class for $9 \mathrm{~h}$. The refractive error in both eyes after low-level laser irradiation on the auricular points for 30 min after class decreased compared with those immediately after class.

From Table 1, the left and right eye refractive error of the control group decreased by $-0.08 \pm 0.03 \mathrm{D}$ and -0.02 $\pm 0.07 \mathrm{D}$, respectively, compared with the values immediately after class. Results of the paired-sample $t$-test indicated no significant difference $(\mathrm{P}>0.05)$. In the experimental group, the refractive error of both eyes significantly decreased (by $-0.12 \pm 0.08 \mathrm{D}$ in the left eye and by $-0.18 \pm 0.01 \mathrm{D}$ in the right eye) compared with the values before laser irradiation. Results of the pairedsample $t$-test indicated a significant difference $(\mathrm{P}<0.05)$.

\section{Eye Accommodation Examination}

In Table 2, we have shown the results of the control group and experimental group in the amplitudes of accommodation (AA) examination. The AA in the control group (without low-level laser irradiation) before and after class and after the 30-min break with eyes closed is shown in Figure 8. The mean AA of the left and right eyes before class decreased by $2.35 \pm 0.38 \mathrm{D}$ and $2.1 \pm 0.01 \mathrm{D}$, respectively, compared with the values immediately after class. Results of the pairedsample $t$-test indicated a significant difference $(\mathrm{P}<0.05)$. The results suggested that the AA of the participants' eyes decreased after the participants attended class for 9 h. Moreover, the mean AA of the left and right eyes after the 30-min break with eyes closed was, respectively, $1.06 \pm$ $0.01 \mathrm{D}$ and $1.05 \pm 0.04 \mathrm{D}$ higher than their AAs immediately after class. Results of the paired sample $t$-test indicated a significant difference $(\mathrm{P}<0.05)$. The results suggested

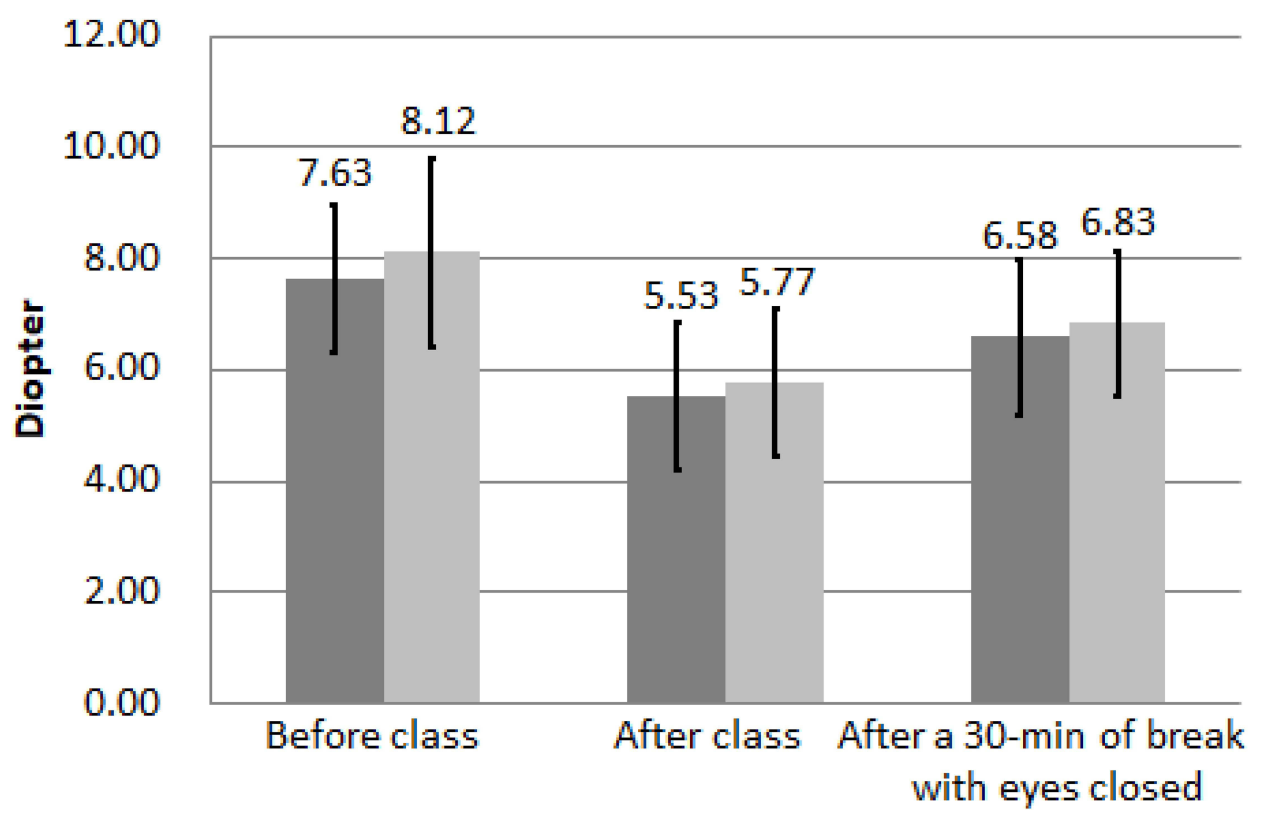

RIGHT EYE — LEFT EYE

Figure 8 AA before and after class and after a 30-min break. 
that the participants' eye AA increased after the 30-min break with eyes closed after they attended class for $9 \mathrm{~h}$.

However, even after the 30-min break after class with their eyes closed, the participants' mean AA in both eyes was lower than that before class. The differences in the AA between the left and right eyes were $1.29 \pm 0.37 \mathrm{D}$ and 1.05 $\pm 0.05 \mathrm{D}$, respectively. These results indicated that after attending 9 hours of class, the participants experienced decreased AA even after the 30-min break with eyes closed.

The AAs of the participants in the experimental group before and after class and after low-level laser irradiation for 30 min are shown in Figure 9. According to Table 2, the mean AAs of the left and right eyes before class were, respectively, $2.35 \pm 0.38 \mathrm{D}$ and $2.1 \pm 0.01 \mathrm{D}$ lower than those after class. Results of the paired-sample $t$-test revealed a significant difference $(\mathrm{P}<0.05)$, suggesting that the participants' AA in both eyes decreased after $9 \mathrm{~h}$ of class. Moreover, the mean AAs of the left and right eyes after lowlevel laser irradiation for $30 \mathrm{~min}$ were, respectively, $2.14 \pm$ $0.08 \mathrm{D}$ and $2.12 \pm 0.02 \mathrm{D}$ higher than those immediately after class. Results of the paired-sample $t$-test indicated significant difference $(\mathrm{P}<0.05)$. The results revealed that the participants' eye AA increased after low-level irradiation for $30 \mathrm{~min}$ conducted after $9 \mathrm{~h}$ of class.

From Table 2, we can note that the mean AAs of the left and right eyes after low-level laser irradiation for 30 min after class were, respectively, $0.05 \pm 0.04 \mathrm{D}$ and 0.05
D higher than those before class. Results of the pairedsample $t$-test revealed no significant difference $(\mathrm{P}>$ 0.05). The AAs before class and after low-level irradiation for $30 \mathrm{~min}$ did not differ considerably, implying that low-level laser irradiation for $30 \mathrm{~min}$ could help recover the AA to a level comparable to that before class.

\section{Discussion}

In the refractive error test, we observed that both the control group and the experimental group of teenagers showed an increase in negative refractive error in both eyes after 9 hours of class, which means that the distance of the farsightedness of the teenagers became shorter after 9 hours of class. As a result, the AA will be lowered. Obviously, we can see that both the control group and the experimental group of teenagers show a decrease in their adjustment range after 9 hours of class. In addition, regardless of the refractive error of the left (right) eye of the control group after 30 minutes of rest with eyes closed, or the refractive error of both eyes of the experimental group after light exposure, the negative refractive error of both eyes decreased, so AA can be expected to increase accordingly.

Finally, in the control group (without laser irradiation), the mean AA of the participants' right and left eyes recovered by $50.0 \%$ and $45.1 \%$, respectively, after the $30-\mathrm{min}$ break. In the laser irradiation group, the mean AA of the participants' right and left eyes recovered by the level before the class, after low-level laser irradiation. Therefore,

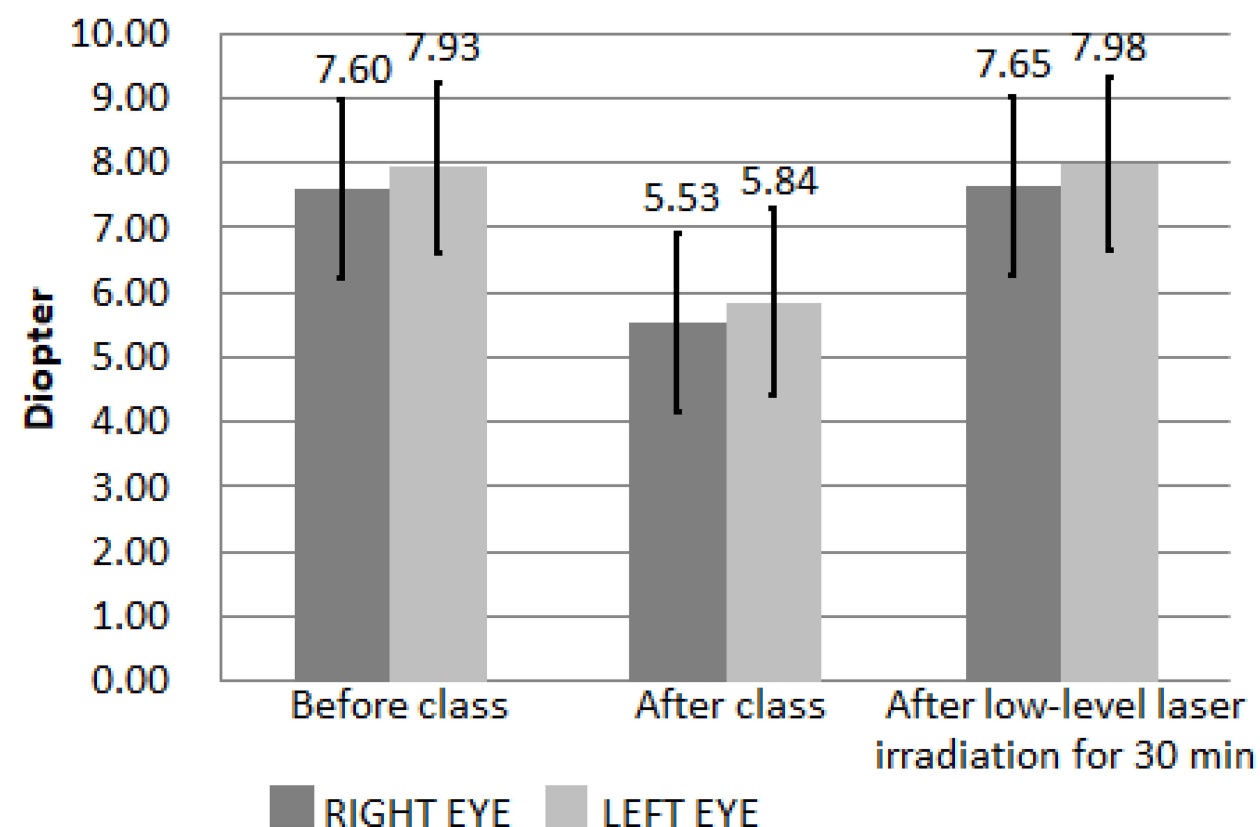

Figure $9 \mathrm{AA}$ before and after class and after low-level laser irradiation for $30 \mathrm{~min}$. 
the second-stage comparison of groups treated with and without low-level laser irradiation on auricular points indicated that the AA of the participants who underwent lowlevel laser irradiation increased, obviously. The effectiveness of the 30-min low-level laser irradiation in AA recovery was higher than that of the 30-min break. According to the description by Sterner et $\mathrm{al}^{19}{ }^{19}$ we compared the Hofstetter's curve of the AA. For teenagers aged 16-18 with myopia after class, our AA results are reduced by about $8 \mathrm{D}$. Therefore, we believe that the participants did reach the level of visual fatigue after 9 hours of class. However, the participants who received low-intensity laser irradiation could indeed achieve the effect of quickly alleviating visual fatigue caused by changes in accommodation.

\section{Conclusion}

This study compared the effects of 30-min low-level laser irradiation at auricular points with that of a 30-min break comprising closing of eyes on eye accommodation. Lowlevel laser irradiation at auricular points restored the eye accommodation faster than did natural closing of the eyes. The results indicated that the effectiveness of low-level laser irradiation was comparable to that of traditional Chinese medicine and thus can be used as an alternative therapy for patients who are unwilling to undergo acupuncture and moxibustion.

The following conclusions were drawn:

(1) Teenagers read books or use consumer electronics for prolonged periods in school environments. Such behavior reduces their eye accommodation, thereby causing visual fatigue and increasing the dioptric power.

(2) Low-level laser irradiation at auricular points and resting with eyes closed can relax visual fatigue caused by changes in accommodation and help regain the original dioptric power. Low-level laser irradiation at auricular points recovered the visual acuity faster than rest with eyes closed over the same duration.

(3) Participants who received low-level laser irradiation at auricular points reported no piercing pain of needlesticks.

In summary, low-level laser irradiation at auricular points can largely benefit people who are afraid of undergoing traditional acupuncture. Low-level laser irradiation at auricular points for eyes can rapidly reverse temporary myopia. Further research can explore acupoints around the eyes. Furthermore, acupuncture therapy is a safe and effective therapeutic method for treating myopia with no adverse effects. Some studies ${ }^{20-22}$ have proposed that stimulation at auricular points is beneficial for comprehensive adjustment functions of various tissues and organs of the body. Therefore, auricular therapy is suitable for clinical use because of its safety, reliability, nontoxicity, lack of side effects, and ease of use.

\section{Ethical Approval}

All the authors' institutions have not established the Institutional Review Board. According to Chapter 2 Article 5 of the Taiwan Human Body Research Act, prior to conducting a research, the principal investigator shall submit the research protocol for review and approval by the Institutional Review Board (hereinafter "IRB"). The review in the preceding Paragraph shall be conducted by the research entity's IRB. Where an entity does not have an established IRB, the review may be conducted by IRB of other entity. In this study, all procedures performed were in accordance with the ethical standards of the Human Research Ethics Committee at National Cheng Kung University (Approval No. NCKU HREC-E-107337-2) and with the 1964 Helsinki declaration and its later amendments or comparable ethical standards.

\section{Informed Consent}

Informed consent was obtained from all patients. The person in Figure 2 is one of authors and provided written informed consent for the image to be published.

\section{Funding}

No external funding sources to declare.

\section{Disclosure}

The authors declare that they have no competing interests.

\section{References}

1. Round R, Litscher G, Bahr F. Auricular acupuncture with laser. Evid Based Complement Alternat Med. 2013; 2013:984763. doi:10.1155/ 2013/984763

2. Mester E. The use of the laser beam in therapy. Orv Hetil. 1966;107 (22):1012-1016.

3. Zhongming L, Wugao C. Quantitative analysis of thermal effect of laser acupuncture and moxibustion. Appl Laser. 1995;15(6):277-279.

4. Chang-chun Z, Xian-ju W, Zi-ru L, Han-ping L, Zhou-yi G, Song-hao L. Laser acupuncture and its information modeling. Chin J Clin Rehabilitation. 2005;9(17):175-177.

5. Whittaker P. Laser acupuncture: past, present, and future. Lasers Med Sci. 2004;19(2):69-80. doi:10.1007/s10103-004-0296-8

6. Zhong-ming L, Zhen-xi Z. Laser acupuncture theory and light dosage selection. Acta Laser Biol Sinica. 2006;15(3):271-277. 
7. Qing-sheng Z, Shi-zhong L. Acupuncture combined with auricular acupressure in treating myopia among 1068 teenagers. Zhongguo Zhen Jiu. 2002;22(12):810.

8. Wang Y, Gao YX, Sun Q, et al. Acupuncture for adolescents with mild-to-moderate myopia: study protocol for a randomized controlled trial. Trials. 2014;15(1):477. doi:10.1186/1745-6215-15-477

9. Wei ML, Liu JP, Li N, Liu M. Acupuncture for slowing the progression of myopia in children and adolescents. Cochrane Database Syst Rev. 2011; (9). Art. No.: CD007842. doi:10.1002/14651858. CD007842.pub2

10. Gao H, Zhang L, Liu J. Auricular acupressure for myopia in children and adolescents: a systematic review. Complement Ther Clin Pract. 2020;38:101067. doi:10.1016/j.ctcp.2019.101067

11. Yachan L, Zhang O, Wenna L, Candong L. Effect of auricular pressing treatment on myopia in children. J Trad Chin Med. 2015;35 (3):281-284. doi:10.1016/S0254-6272(15)30098-4

12. Yu Z, Wang X, Zhao X, Li D, Duan J. The effectiveness and safety of acupuncture for patients with myopia: a protocol for a systematic review and meta-analysis. Medicine. 2020;99(23):e20410. PMID: 32501987; PMCID: PMC7306361. doi:10.1097/ MD.0000000000020410

13. Chun-nian L. Preliminary experience of myopia treated using eye points in acupuncture through intradermal needle therapy. Chin J Med. 1988;23(5):29.

14. Wei L. Cowherb seed auricular acupressure in treating myopia among 70 teenagers. Henan Trad Chin Med. 2012;32(8):1030-1031.
15. Sheedy JE, Hayes JR, Engle J. Is all asthenopia the same? Optom Vis Sci. 2003;80(11):732-739. doi:10.1097/00006324-200311000-00008

16. Gowrisankaran S, Sheedy JE, Hayes JR. Eyelid squint response to asthenopia-inducing conditions. Optom Vis Sci. 2007;84(7):611-619. doi:10.1097/OPX.0b013e3180dc99be

17. Jaschinski-Kruza W. Visual strain during VDU work: the effect of viewing distance and dark focus. Ergonomics. 1988;31 (10):1449-1465. doi:10.1080/00140138808966788

18. Saito S, Taptagaporn S, Salvendy G. Visual comfort in using different VDT screen. Intl J of Hum Comput Interact. 1993;5(4):313-323. doi:10.1080/10447319309526071

19. Sterner B, Gellerstedt M, Sjöström A. Accommodation and the relationship to subjective symptoms with near work for young school children. Ophthalmic Physiol Opt. 2006;26(2):148-155. doi:10.1111/j.1475-1313.2006.00364.x

20. Bruce-Low SS, Cotterrell D, Jones GE. Heart rate variability during high ambient heat exposure. Aviat Space Environ Med. 2006;77 (9):915-920.

21. Xia Y. Survey of acupuncture on chingming point in treating myopia among teenagers. J Clin Opthalmol. 2001;9(2):151-152.

22. Zhi Z, Yuhong Z. Auricular acupressure in treating myopia among 146 teenagers and observation of therapeutic efficacy. Yunnan J Trad Chin Med Mat Med. 2000;21(2):41-42.
Clinical Ophthalmology

\section{Publish your work in this journal}

Clinical Ophthalmology is an international, peer-reviewed journal covering all subspecialties within ophthalmology. Key topics include: Optometry; Visual science; Pharmacology and drug therapy in eye diseases; Basic Sciences; Primary and Secondary eye care; Patient Safety and Quality of Care Improvements. This journal is indexed on PubMed
Dovepress

Central and CAS, and is the official journal of The Society of Clinical Ophthalmology (SCO). The manuscript management system is completely online and includes a very quick and fair peer-review system, which is all easy to use. Visit http://www.dovepress.com/ testimonials.php to read real quotes from published authors. 